\title{
Experimental Proof of Mass in Photon
}

\author{
Narendra Swarup Agarwal \\ 704 HUDA CGHS Limited, Gurgaon, India \\ Email: nsagarwal@gmail.com
}

Received 3 March 2015; accepted 17 April 2015; published 21 April 2015

Copyright (C) 2015 by author and Scientific Research Publishing Inc.

This work is licensed under the Creative Commons Attribution International License (CC BY).

http://creativecommons.org/licenses/by/4.0/

(c) (i) Open Access

\begin{abstract}
The present work deals with the mass of photon which creates a force at the contact point of reflection resulting in slight deviation of photon from its path after reflection. Only the mass of photon can cause deviation due to inertial force. If photon has no mass, it cannot deviate. It is also found that this deviation of photon depends on the angle of polarization plane of laser light with respect to reflecting mirror. Such variation in the angle of deviation by the plane of polarization proves that the mass of photon is neither in the centre of photon nor distributed uniformly but located off-centre (away from the centre of photon). This work proves that photon has mass and the most important part of this work is to discover that the mass of the photon is located off-centre (away from the centre of photon). This discovery explains Wave Particle Duality of electromagnetic radiations.
\end{abstract}

\section{Keywords}

Photon, Mass, Off-Centre, Reflection \& Deviation

\section{Introduction}

Photon is a particle and displays dual nature both of particle as well as wave. The wave particle duality of photon has been accepted in quantum mechanics. However, Einstein [1] did not accept wave-particle duality and wrote:

"This double nature of radiation (and of material corpuscles) is a major property of reality, which has been interpreted in quantum mechanics in an ingenious and amazingly successful fashion. This interpretation, which is looked upon essentially as final by almost all contemporary physicists appears to me as only a temporary way out”.

To find the solution of wave particle duality solar system is considered, it is estimated that the mass of the Sun is $>99.86 \%$ of the mass of the whole solar system while the Sun occupies only about $10^{-10}$ time volume of the solar system. The Sun is not in the centre but it is on one of the epicentre of the elliptical path of the planets.

Similarly in case of atom, it is known that the nucleus has mass $>99.9 \%$ of the mass of the whole atom but 
occupies only about $10^{-10}$ times the volume of the atom.

New Quantum Theory [2], developed in the year 2012 is based on the analogy of solar system \& atom to explain wave particle duality of photon. This theory is based on the hypothesis that a photon has a nucleus of mass with the nucleus located off-centre in the photon. Nearly all the mass of the photon is located in its nucleus similar to the solar system and the atoms. This hypothesis can explain not only Wave Particle Duality but also other phenomena of electromagnetic radiations.

The experiments are conducted to confirm the hypothesis of New Quantum Theory "whether photon has mass or zero mass". Polarized laser light is reflected on plain surface coated mirror. The results of the experiments are astonishing and given below:

- The photons of polarised laser light on reflection slightly deviate from their path after reflection.

- The angle of deviation of photons on reflection depends on the angle of plane of polarization of laser light with respect to the plane of reflecting mirror.

- The angle of deviation increases with frequency of laser light.

- The angle of reflection is slightly different than the angle of incidence.

The deviation of the photons of polarized laser light on reflection is due to the force created by the mass of the photon at the contact point of reflection. Force can only be created if photon has mass. A photon of zero mass cannot create any force at the contact point of reflection and will not deviate. The mass of spinning photon creates the force to turn at the contact point of reflection resulting in the deviation of photon and change the direction of photon.

Further the change in the angle of deviation of photons with variation in the plane of polarization indicates that the force created depends on the position of the nucleus of mass in spinning photon with respect to the contact point of reflection. If the photon has either uniformly distributed mass or located in the centre of photon, there will be no variation in the angle of deviation of photon with change in the plane of polarization. This work finds that the mass in the photon is neither uniformly distributed nor it is in the centre of the photon but the mass of photon is located off-centre (away from the centre).

The existence of mass in photon is also indicated by the followings:

- It is already known that the light from a star bends while passing near the sun due to the gravitational pull of the sun. The light from star bends due to the universal gravitational force which is proportional to the mass of photon and the mass of sun.

- D. D. Ryutov in article "Using plasma physics to weigh the photon" [3] has mentioned the following:

"The currently accepted value for the upper bound for the photon mass, $m_{p h}$, is 22 orders of magnitude less than the electron mass. As the mass $m_{p h}$ is so incredibly small, it has essentially no effect on atomic and nuclear physics".

\section{Experiment}

The items used in the experiments are listed below:

1) Red laser light of wavelength $632.8 \mathrm{~nm}$ (He-Ne, $2 \mathrm{~mW}$ of Melles Griot, USA).

2) Green laser light of wavelength $532 \mathrm{~nm}$.

3) Polariser.

4) Plain surface coated mirror ( $\lambda / 10 \mathrm{~nm}$ of Optiregion, New Delhi) of size approx. $25 \mathrm{~mm} \times 25 \mathrm{~mm}$.

5) White screen of size approx. 1 meter $\times 1$ meter installed approx. 3 meters from plain surface coated mirror.

The top view of the experimental set up is shown in Figure 1. The laser light with polarizer is mounted at approx. $15^{\circ}$ angle from the horizontal. The polarized laser light falls on the horizontally placed plain surface coated mirror on the table. The laser light after reflection falls on the screen.

The angle of polarization planes of $632.8 \mathrm{~nm}$ or $532 \mathrm{~nm}$ laser lights are initially kept in vertical plane at $0^{\circ}$ degree. The position of reflected laser light from plain mirror on the screen is recorded. The plane of polarization is changed from $0^{\circ}$ to $30^{\circ}, 60^{\circ}$ and $90^{\circ}$ from the vertical plane. The deviation of photons of laser light is recorded on the screen and angle of deviation is calculated. The deviation of photons and angle of reflected light are given in the Table 1 and Table 2.

The experiments can be repeated in any physics lab using different wavelengths of polarized laser light of narrow beam.

The orientation of photon in vertical and horizontal planes of polarization are shown in Figure 2 and Figure 3 


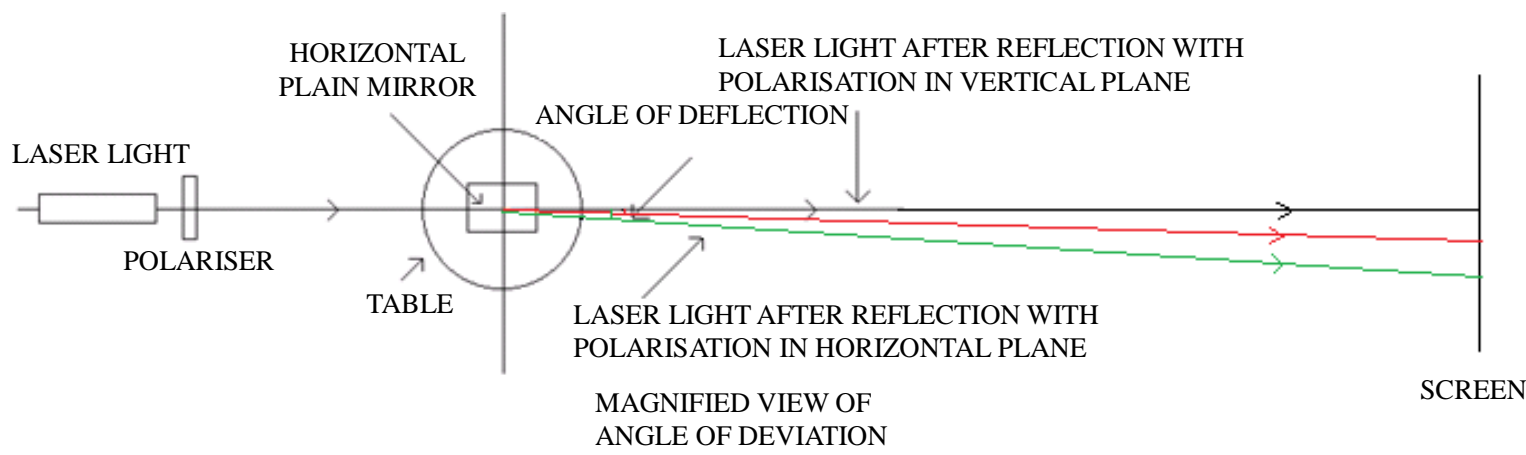

Figure 1. Top view of deviation of photons of laser lights due to the mass of photon at the point of reflection (the angle of deviation of polarized laser light is magnified in above diagram for the purpose of explanation only).

Table 1. Experiment with red laser light of $632.8 \mathrm{~nm}$ wavelength.

\begin{tabular}{ccccc}
\hline Sr. No. & $\begin{array}{c}\text { Angle of polarization } \\
\text { from vertical }\end{array}$ & $\begin{array}{c}\text { Deviation of photons on projector } \\
\text { screen at distance } 3050 \mathrm{~mm}\end{array}$ & $\begin{array}{c}\text { Total deviation of laser light } \\
\text { from vertical polarization plane }\end{array}$ \\
\hline 1 & Degree & mm & Minutes & Seconds \\
2 & 0 & 0 & 0 & 0 \\
3 & 30 & 0.5 & 0 & 33 \\
4 & 60 & 1.3 & 2 & 4 \\
\hline
\end{tabular}

Table 2. Experiment with green laser light of $532 \mathrm{~nm}$ wavelength.

\begin{tabular}{|c|c|c|c|c|}
\hline \multirow[t]{2}{*}{ Sr. No. } & \multirow{2}{*}{$\begin{array}{c}\text { Angle of polarization } \\
\text { from vertical } \\
\text { Degree }\end{array}$} & \multirow{2}{*}{$\begin{array}{c}\text { Deviation of photons on projector } \\
\text { screen at distance } 2950 \mathrm{~mm} \\
\mathrm{~mm}\end{array}$} & \multicolumn{2}{|c|}{$\begin{array}{l}\text { Total deviation of laser light } \\
\text { from vertical polarization plane }\end{array}$} \\
\hline & & & Minutes & Seconds \\
\hline 1 & 0 & 0 & 0 & 0 \\
\hline 2 & 30 & 0.8 & 0 & 55 \\
\hline 3 & 60 & 1.7 & 2 & 08 \\
\hline 4 & 90 & 3.2 & 3 & 50 \\
\hline
\end{tabular}

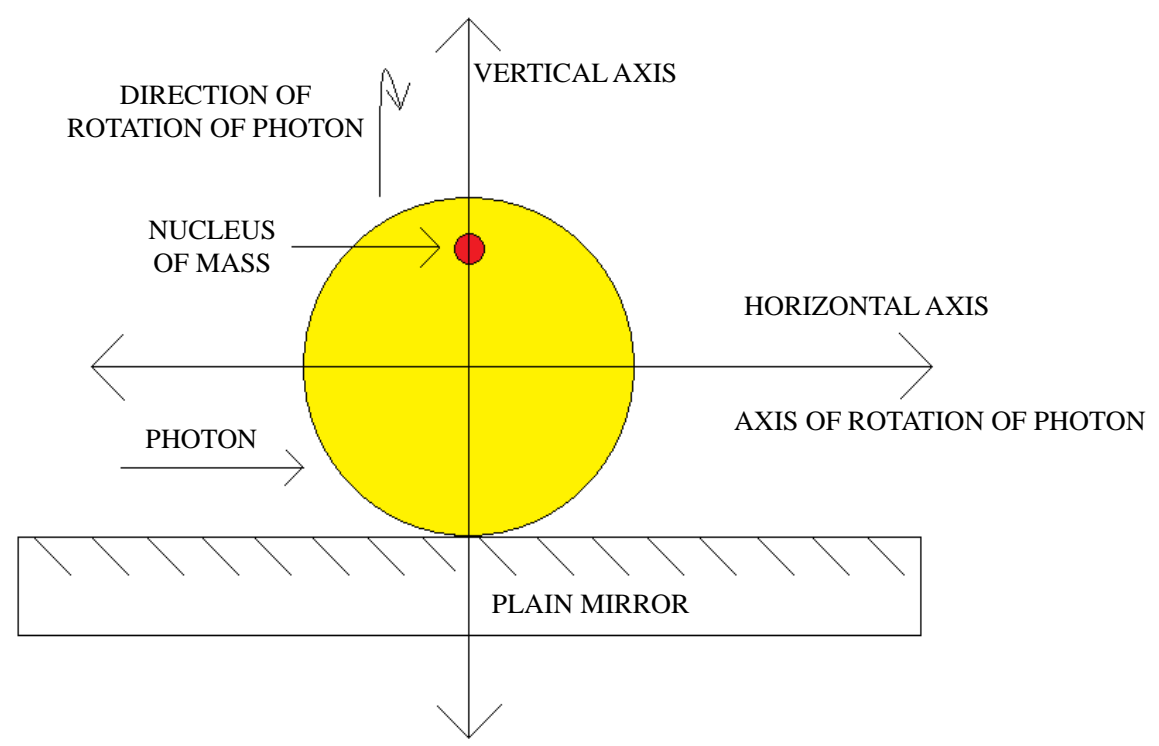

Figure 2. Photon with vertical plane of polarization with no horizontal deviation of photon on reflection. 


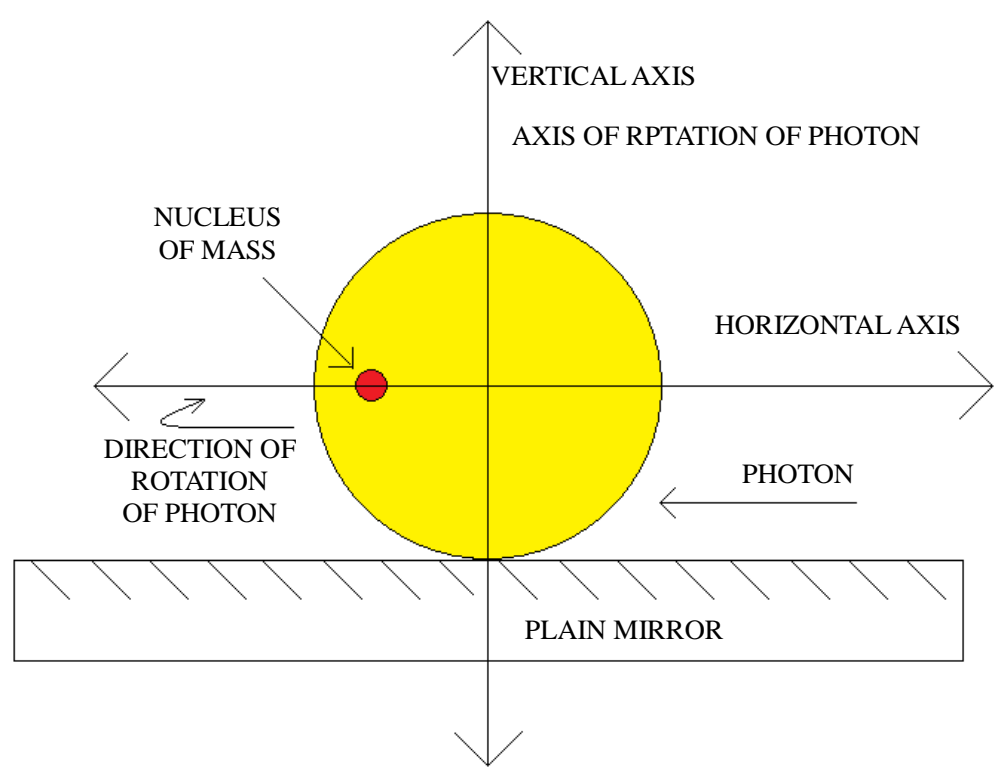

Figure 3. Photon with horizontal plane of polarization with maximum deviation of photon in horizontal direction after reflection.

respectively. The simulation of reflection of polarized laser light on plain surface coated mirror at $0^{\circ}$ to $30^{\circ}, 60^{\circ}$ and $90^{\circ}$ from vertical plane is shown in Figure 4.

\section{Wave Particle Duality}

As proved by the experiments, photon has mass located off-centre (away from the centre of photon). Photon is a spinning particle moving with velocity of light. Therefore following momentums act on a photon:

- Linear momentum in the direction of travel of photon, this is always constant in one direction.

$$
\text { Linear momentum }=M_{p} C
$$

- Angular momentum (with respect to the centre of photon) due to the spin of photon with its mass located off-centre. The direction of this varies continuously from $0^{\circ}$ to $360^{\circ}$ with the spin of photon.

$$
\begin{aligned}
\text { Angular momentum } & =I_{n} \omega \\
& =M_{n} r^{2} \omega \\
& =M_{n} r^{2} 2 \pi f \\
& =2 \pi f M_{n} r^{2}
\end{aligned}
$$

where $M_{p}$ : Total Mass of Photon;

$M_{n}$ : Mass of Nucleus of Photon;

c: Velocity of Photon;

$I_{n}$ : Moment of Inertia of Nucleus of mass;

$r$ : Distance of Nucleus of mass from centre of Photon;

$f:$ Frequency of the Photon;

$\omega$ : Angular Velocity.

Wave Particle Duality is explained by these experiments discovering the existence of mass in the photon located away from the centre of photon. The angular momentum due to the mass of photon located off-centre (away from the centre of photon) is responsible for the up and down movements of the photon from its centre line path to form a sinusoidal wave instead of travelling in straight line.

As the photon spins clockwise the direction of angular momentum due to the mass of the photon continuously changes clockwise. At position 1 of photon in Figure 5 the direction of angular momentum is $90^{\circ}$ (Y-Axis); this moves the photon upward from its horizontal centre line path (X-Axis). As the photon further spins clockwise, the direction of angular momentum changes from $90^{\circ}$ to $45^{\circ}$ at position 2 of the photon and so on. The resultant vector of linear momentum and angular momentum keep on changing with the spin of the photon, this moves 


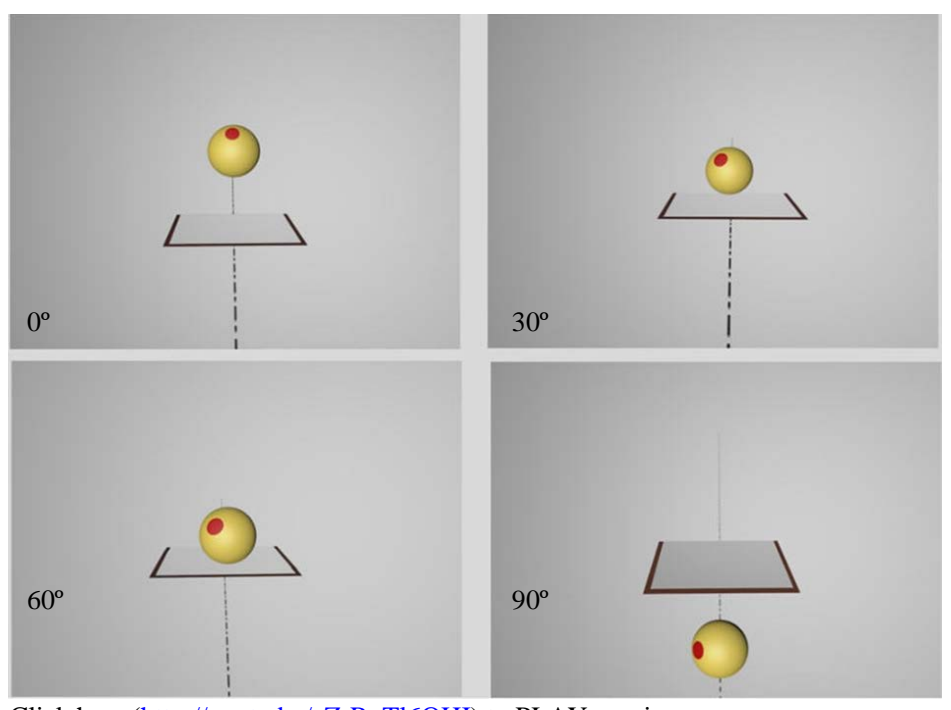

Click here (http://youtu.be/eZrRgTl6OHI) to PLAY movie

Figure 4. Deviation of photons on reflection on plain surface coated mirror. The deviation of photon increases with increase in angle of polarization from $0^{\circ}, 30^{\circ}, 60^{\circ} \& 90^{\circ}$ from the vertical plane. The mirror also shows the image of photon.

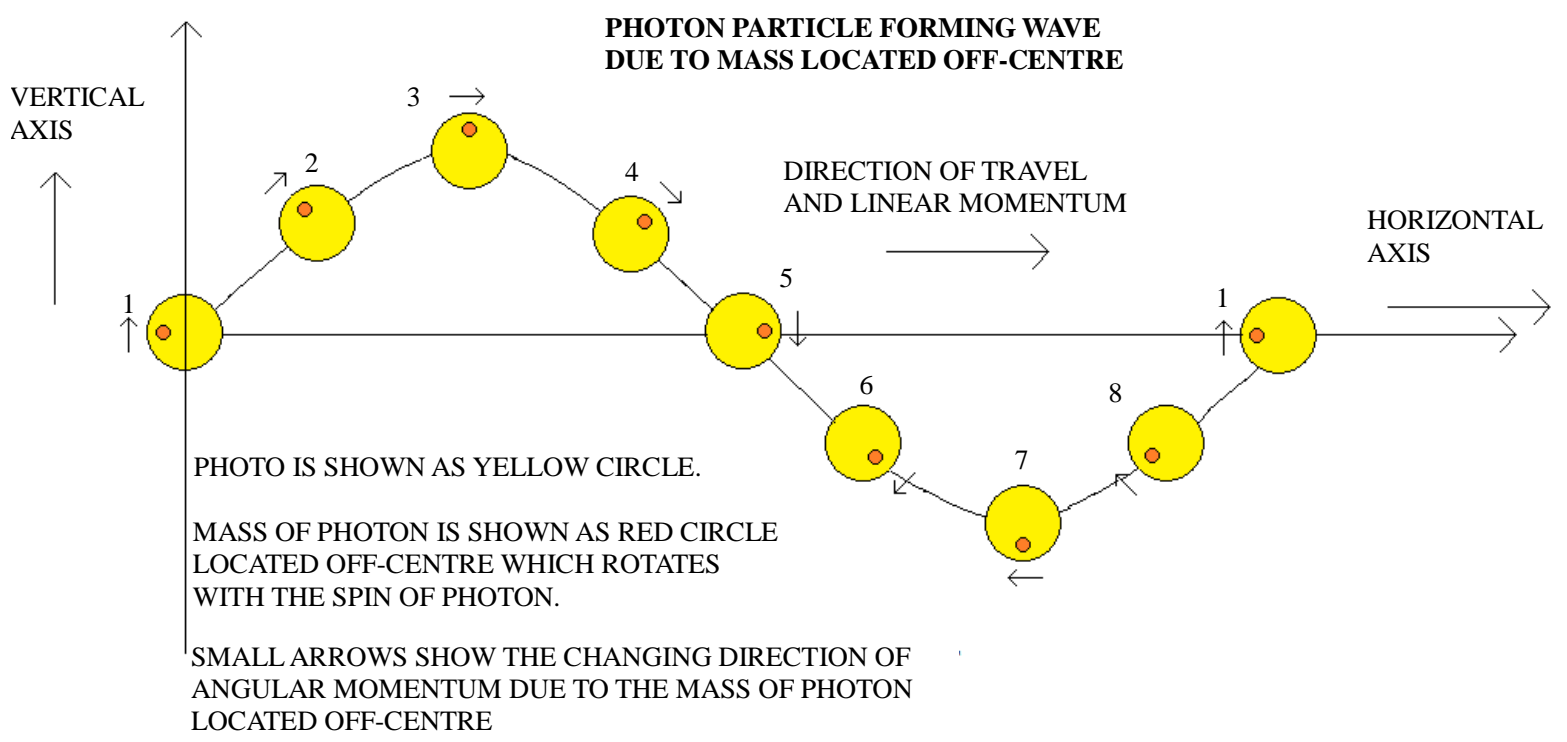

Figure 5. Sinusoidal wave formed by photon particle due to the mass located off-centre.

the photon up and down in Y-Axis while the photon is travelling in X-Axis. The formation of a wave by a photon in vertical polarization plane is shown in Figure 5, the directions of angular momentums due to the mass located off-centre in photon at $45^{\circ}$ intervals of rotation of photon are shown by arrows.

This explains how a photon always travels in the path of a wave. Photon even being a particle always travels as a wave and displays Wave Particle Duality. Simulation of three different wavelengths of visible light displaying the wave formation by photon particles due to their mass located off-centre is shown in Figure 6 .

\section{Analysis \& Conclusion}

1) A photon deviates from its path by the force created at the contact point of plain surface coated mirror due to the mass of the photon. Such deviation is possible only if photon has mass.

\section{- Photon has mass.}

2) The angle of deviation of photon increases when the plane of polarization changes from vertical plane to 


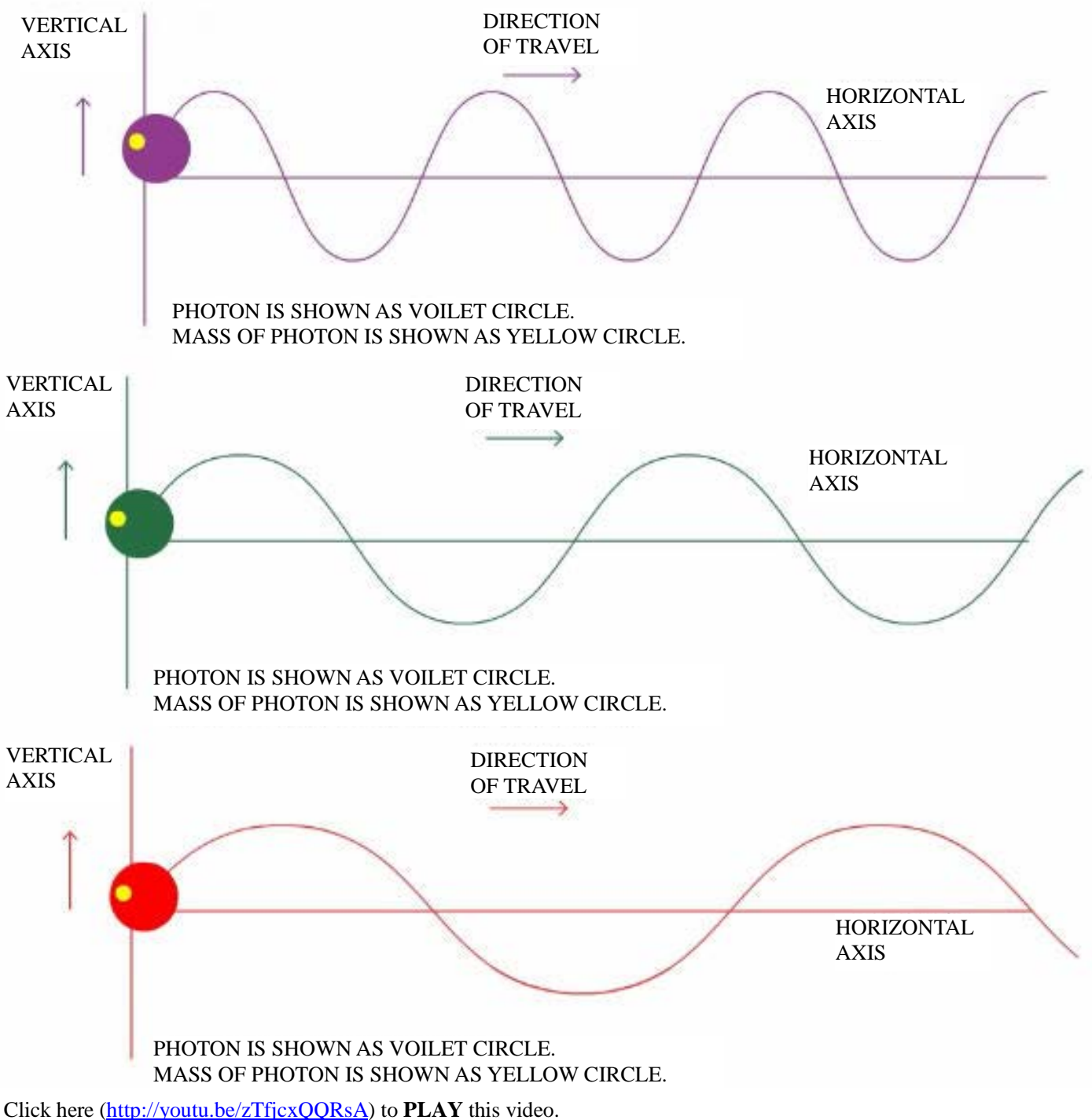

Double Click here (http://youtu.be/zTfjcxQQRsA) to PLAY this video.

Figure 6. Photons of different wavelengths forming sinusoidal waves due the mass of photon located off-centre.

wards the horizontal plane with reflecting mirror in horizontal position. On changing the plane of polarization towards horizontal, the horizontal distance between the mass of photon and the contact point of reflection increases. This increases horizontal vector of angular momentum resulting in higher deviation of the photon. The increase in angular momentum is not possible if the mass of photon is either uniformly distributed or in the centre of photon.

- The mass of photon is neither distributed uniformly nor in the centre, but is located off-centre (away from the centre in the photon).

3) The photons of green laser $(532 \mathrm{~nm})$ deviate more than the photons of red laser (632.8 nm). Higher frequency generates higher angular momentum due to higher angular velocity resulting in more deviation of the photon.

- Higher the frequency of photons, higher is the deviation of photon on reflection.

4) The mass of photon located off-centre generates angular momentum with direction changing from 0 to $360^{\circ}$ in one spin or sinusoidal cycle or wavelength of photon. This angular momentum moves the photon away 
and close from its centre line path in the plane of polarization to form a sinusoidal wave path by a particle of photon.

- Photon even being a particle always moves in the path of sinusoidal wave. Being a particle, photon displays particle nature and by virtue of its always travelling in the path of a wave, photon also displays wave nature. Therefore photon exhibits Wave Particle Duality.

The experimental proof of mass in photon and also the proof of mass being located off-centre in the photon can explain not only the wave particle duality but also many other phenomena of electromagnetic radiations.

\section{References}

[1] Selleri, F. (1990) Quantum Paradoxes and Physical Reality. Kluwer Academic Publishers, The Netherlands.

[2] Agarwal, N.S. (2012) Indian Journal of Science and Technology, 5, 3612-3617.

[3] Ryutov, D.D. (2007) Plasma Physics and Controlled Fusion, 49, B429.

http://dx.doi.org/10.1088/0741-3335/49/12B/S40 